\title{
K fragments
}

\author{
S. Wycech ${ }^{a}$, A.M. Green ${ }^{b}$

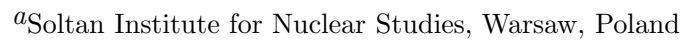 \\ ${ }^{b}$ Helsinki Institute of Physics, University of Helsinki, Finland
}

The $K N N N$ bound states recently discovered at KEK are studied. It is shown that the $\Lambda(1405)$ and $\Sigma(1385)$ resonant states coupled to the $K N$ system may generate an attraction strong enough to form such bound states.

\section{Introduction}

The bound $K N N N$ states, discovered at KEK [1], open up a new sector of strange nuclei. The state found in the $K^{-}$pnn system is bound by $195 \mathrm{MeV}$. A related $K^{-} p p n$ state bound by some $20 \mathrm{MeV}$ less is reported to this conference [2]. These states live fairly long and decay into a hyperon and two nucleons. Nuclear states of kaons were expected, since early studies of kaonic atoms indicated that the nuclear potential for $K^{-}$mesons is attractive, see review [3]. However, the $(K, \pi)$ conversion is very rapid and such states would be difficult to detect due to large $(\approx 100 \mathrm{MeV})$ widths. One way to form states of longer lifetime would be to block the main decay channels. That may happen if the $K^{-}$is bound very strongly. Such a possibility was indicated to exist in the nuclear matter situation, [4]. Akaishi and Yamazaki predicted the existence of narrow states also in light nuclei such as He, [5], and this prediction led to the experiment of Ref. [1]. In both cases the mechanism of attraction was attributed to the resonant $\Lambda(1405) I=0$ state coupled to $K^{-} p+K^{0} n$.

The actual experiment [1] shows that the attraction due to the $\Lambda(1405)$ apparently plays an important role but is not strong enough to generate the binding as observed [6]. Both predictions [4] and [5] were based on a sizeable proton component in the nucleus and that is not the case in the $K^{-}$pnn system. One line of explanation is given at this conference, in terms of a contraction of the nuclear system [7]. Here, it is shown that, under fairly standard nuclear conditions another possibility exists. Two resonant states $\Lambda(1405)$ and $\Sigma(1385)$ coupled to the $K N$ system may generate the attraction required to produce the strong binding. This happens under two basic conditions

- the $\Lambda(1405)$ and $\Sigma(1385)$ in a nuclear medium are located above the $K N$ threshold.

- the binding of $K^{-}$is strong enough to block the main $\pi \Sigma$ and $\pi \Lambda$ decay modes. 
Such conditions may be fulfilled also in heavier nuclei, but the state generated with this interaction will be similar everywhere. The meson is bound in a restricted small area in a region of the highest neutron density.

\section{The origin of $K^{-}$attraction to nuclei}

Let the $K N, I=0 S$-wave scattering amplitude be described by a simple resonance formula

$$
f_{o}=\frac{\gamma_{o}^{2}}{E_{K N}-E_{o}+i \Gamma_{o} / 2},
$$

where $E_{K N}$ is the energy in the $K N$ channel and $E_{o}$ is the $\Lambda(1405)$ energy. This amplitude is normalised to the scattering length at the threshold. For kaons in a nuclear medium it generates an optical potential $U_{o}=2 m_{K} V_{o}$ with

$$
V_{o}(r)=\frac{4 \pi}{2 \mu_{K N}} \rho(r) f_{o},
$$

where $\mu_{K N}$ is the $K N$ reduced mass and $\rho(r)$ is the nuclear density. The resonant situation produces an attractive potential when $E_{K N}-E_{o}<0$ and a repulsive one otherwise. One question to settle is the value of the actual energy of the resonance in the nuclear medium. Emulsion studies of $K^{-}$absorption at the nuclear surface indicate an upward shift of some $10 \mathrm{MeV},[11]$. Nuclear matter calculations generate an upward shift of some 50-100 MeV at central densities as a result of Pauli blocking, [9], [10],[11]. This creates the $E_{K N}-E_{o}<0$ situation which results in an attractive potential.

The other resonance, $\Sigma(1385)$, coupled to the $K N I=1, P$ wave is located far below the threshold. In atomic states $\Sigma(1385)$ seems to play little role. However, in deeply bound states it becomes the dominant factor. The scattering amplitude $f_{\Sigma}=2 \mathbf{p} \mathbf{p}^{\prime} A_{\Sigma}$ is given by a resonant-like volume

$$
A_{\Sigma}=\frac{\gamma_{\Sigma K N}^{2}}{E_{K N}-E_{\Sigma}+i \Gamma_{\Sigma} / 2}
$$

where $\mathbf{p}, \mathbf{p}^{\prime}$ are the relative momenta and $\gamma_{\Sigma K N}$ is a coupling constant. The $\Sigma(1385)$ width is composed of three terms $\Gamma_{\Sigma} / 2=\Sigma_{i} p_{i}^{3} \gamma_{i}^{2}\left(p_{i}^{2}\right)$ and the sum extends over the channels : $\pi \Sigma, \pi \Lambda, K N$. The resonance decays mostly $(0.87 \%)$ to the $\pi \Lambda$ channel and an experimental width of $36 \mathrm{MeV}$ gives $\gamma_{\Sigma \pi \Lambda}^{2}$. The corresponding coupling to the $K N$ channel is related by $\mathrm{SU}(3) \gamma_{\Sigma K N}^{2} / \gamma_{\Sigma \pi \Lambda}^{2}=2 / 3$, while an experimental ratio of $0.51 \pm 0.18$ has been obtained in $K^{-} \mathrm{D}$ capture [14]. Inside the nuclear medium $f_{\Sigma}$ yields an optical potential

$$
U_{G}=\overleftarrow{\nabla} U_{\Sigma}\left(E_{K N}\right) \vec{\nabla}
$$

where $U_{\Sigma}=\left[2 m_{K} 4 \pi /\left(2 \mu_{K N}\right)\right] 2 \rho A_{\Sigma}$. It produces a dramatic effect on the kinetic energy of the meson. The dispersion law in nuclear matter becomes

$$
E_{K}^{2}-m_{K}^{2}=p^{2}\left[1+U_{\Sigma}\left(E_{K N}\right)\right]+U_{o},
$$


where $E_{K}=m_{K}-E_{B}$. For energies $E_{K N}$ close to, but less than $E_{\Sigma}$, the $U_{\Sigma}$ term may dominate the kinetic energy and make negative the $p^{2}$ term in the dispersion formula (5). That happens for energy separations $\left|E_{K N}-E_{\Sigma}\right|<140 \mathrm{MeV}$ at central nuclear densities. In the nuclear matter case it may generate a quasi-collapse which, however, is removed by the resonance denominator. An increased binding generates larger $E_{K N}-E_{\Sigma}$ and a weaker attraction. At the end a finite saturation energy is obtained. The latter depends on the position of $E_{\Sigma}$ in the nuclear medium. An experiment reported at this meeting [8] indicates that it stays at its free value.

\section{The $K^{-} N N N$ system}

In the $K^{-}$pnn situation the $\Lambda(1405)$ may be formed on the proton. Following the nuclear matter calculations we use an upward shift of some $40 \mathrm{MeV}$ for the position $E_{o}$. The $\Sigma(1385)$ is formed more frequently as it involves mostly neutrons and also $E_{\Sigma}$ is apparently not modified [8]. With the $K^{-}$bound by some $180 \mathrm{MeV}$ the relative separation of the $K N$ threshold and the $\Lambda(1405)$ position amounts to $190 \mathrm{MeV}$ and an extrapolation of the scattering amplitudes to this region is necessary. The $K$-matrix parametrization of A.Martin[12] $\left(K_{N N}=-1.65 \mathrm{fm}, R_{N N}=0.18 \mathrm{fm}\right)$ is used here, but it is supplemented by a separable model of Ref.[13] to obtain a smooth subthreshold extrapolation. This procedure generates the $I=0$ scattering amplitude of $f_{0}=1.4 \mathrm{fm}$, a fairly standard result in this energy region. As the energy of $K N$ is so low, the pionic decay channels are closed and the scattering amplitudes are real. For AMG the $K^{-} n, I=1$ amplitude the solution from Ref. [12] is used $\left(K_{N N}=1.07 \mathrm{fm}\right)$ which produces $f_{1}=0.34 \mathrm{fm}$. These values generate the potential $V_{o}=-105 \mathrm{MeV}$ ( $-130 \mathrm{MeV}$ for $K^{-} p p n$ ) for a $K^{-}$at the centre of the tritium nucleus. An additional, and in fact dominant, attraction comes from the $\Sigma(1385)$. We look for the variational solution for the kaonic energy level, $\epsilon=E_{K}^{2}-m_{K}^{2}$,

$$
\epsilon=\operatorname{Min} \int d \mathbf{r} \Psi(r)\left[p^{2}+\overleftarrow{\nabla} U_{\Sigma}\left(E_{K N}\right) \vec{\nabla}+U_{o}\right] \Psi(r)
$$

The results are given in the Table 1 with details of the test function $\Psi$ being found in $[15]$.

Table 1: The binding energies $E_{B}$, in $\mathrm{MeV}$, of the $K^{-}$pnn and $K^{-} p p n$ systems. The first line is based on the $S U(3)$ value for the $K N \Sigma(1385)$ coupling. In the second line this coupling is enhanced by $20 \%$.

\begin{tabular}{lll}
\hline$\gamma_{\Sigma K N}^{2} / \gamma_{\Sigma \pi \Lambda}^{2}$ & $K^{-} p n n$ & $K^{-} p p n$ \\
$2 / 3$ & 164 & 147 \\
$1.2^{*} 2 / 3$ & 187 & 167 \\
\hline
\end{tabular}

In summary:

- The $\Lambda(1405)$ and $\Sigma(1385)$ states coupled to the $K N$ system may generate the strong binding of $K^{-}$mesons, as is observed. Such states, under normal nuclear density tend to be localised close to the nuclear centres to maximize the P-wave attraction. 
- This model requires obvious refinements (1) a better knowledge of the extrapolated scattering amplitudes, (2) the value of the $\gamma_{\Sigma K N}$ coupling, (3) corrections for NNN excitations.

- This model stresses the strengths of the $K^{-} n$ interaction. The search for $K^{-} n n$, $K^{-} n n n$ and other objects of neutron excess could be helpful. This project is financed by the European Community Human potential Program HPRN-2002-00311 EURIDICE.

\section{References}

[1] T. Suzuki et al., Phys.Lett. B597(2004)263.

[2] M. Iwasaki, this conference

[3] C. J. Batty, E. Friedman and A. Gal, Phys. Rep. 287(1997)385

[4] S. Wycech, Nucl.Phys. A450(1986)399c

[5] Y. Akaishi and T. Yamazaki,

Phys.Rev.C65(2002)044005

[6] W. Weise, this conference

[7] Y. Akaishi, this conference

[8] N. Herrmann, this conference

[9] M. Alberg, E. Henley and L. Wilets, Ann.Phys.NY.96(1976)43

[10] S. Wycech, Nucl.Phys. B28(1971)541

[11] L. R. Staronski et al., J.Phys. G13(1987)1387

[12] A. D. Martin, Nucl.Phys. B179(1981)33

[13] W. Krzyzanowski et al., Acta Phys.Pol.B6(1975)259

[14] O. Braun, Nucl.Phys. B129(1977)1

[15] S. Wycech and A.M. Green, nucl-th/0501019 